\title{
Piper betle leaves extract adversely affect motility of Pseudomonas aeruginosa
}

\author{
S.A.M.Sedek ${ }^{1 *}$,M.A.Arifin ${ }^{1,2}$ and M.S.A.Munaim ${ }^{1,2}$ \\ ${ }^{I}$ Faculty of Chemical and Processes Engineering Technology, Universiti Malaysia Pahang, 26300, Kuantan, \\ Pahang, Malaysia \\ ${ }^{2}$ Earth Resources and Sustainability Centre (ERAS), Universiti Malaysia Pahang, 26300, Kuantan, Pahang, \\ Malaysia \\ *Email: saiedatul@gmail.com
}

\begin{abstract}
Pseudomonas aeruginosa is an opportunistic pathogen that orchestrate its disease manifestation via a complex communication system known as quorum sensing (QS). Under the regulation of this complex network of QS system, is a myriad of virulence factors including the flagella and pili that contribute to its motility. To establish an infection in a host, $P$. aeruginosa must first attach itself to host cells with the assistance of flagella and pili and it would be a mere impossible for $P$. aeruginosa to initiate and establish an infection in the host without these features. In this study, we investigated the motility of $P$. aeruginosa under the influence of Piper betle leaves extract by growing the bacteria on a semi solid medium and compared the growth spread of the pathogen against the untreated sample. There are three types of motilities that were being investigated, which were swimming, swarming and twitching. In all three assays, a fraction of ethyl acetate extract of $P$. betle leaves were found to reduce the motility of $P$. aeruginosa with greatest defect in swimming at more than $80 \%$ reduction as compared to the untreated sample. Meanwhile, the fraction partially decreased the swarming and twitching of $P$. aeruginosa by $58 \%$ and $50 \%$ reduction respectively, in comparison to the untreated sample. The exhibited defect in the motility of $P$. aeruginosa proves the anti-QS property of the fraction of ethyl acetate extract of $P$. betle leaves and could be developed as a potential therapeutic agent against the pathogen.
\end{abstract}

Indexed Terms- Motility, Pseudomonas aeruginosa, Piper betle, quorum sensing

\section{INTRODUCTION}

Recent years have witnessed intensive searches for alternative medicine to fight against bacterial infection as opposed to antibiotics. This is due to excessive cases of antibiotic- and multidrug-resistance bacteria in the clinical setting [1]. One of the approaches is by targeting the quorum sensing (QS) system of the bacteria. With a great deal of information on the QS system, researchers have explore the possibilities of hindering the communication network in QS bearing-bacteria without affecting its physiology which should restrain the pathogen from acquiring resistance against the agent. Such approach is better known as anti-QS property and theoretically, disrupting the QS system would block the system from being activated and thus disabling the pathogen from even initiating the infection processes. A number of bioactive compounds isolated from plants, such as ajoene, iberin and protoanemonin, have been demonstrated to possess an anti-QS activity against $P$. aeruginosa $[2,3,4]$.

An opportunistic pathogen like $P$. aeruginosa requires an intricate survival system to endure the harsh environment and to consequently establish an infection in the host. Hence, they are armed with the complex QS system that operates based on the density of the auto-inducer (AI) molecules that act as a regulatory mechanism. A threshold concentration of these AIs would trigger cascades of signaling pathways leading to the transcriptional expression of a range of target genes including the virulence genes to initiate, colonize, and disseminate the infection in a host [5]. These virulence proteins include flagella and type IV pili for the motility of $P$. aeruginosa, toxins such as pyocyanin and exotoxin A, 
and iron-scavenging proteins such as pyochelin, pyoverdin and proteases $[6,7,8]$. P. aeruginosa is also capable of evading the action of host's immune defense by means of elastase and alkaline protease [9]. While the production of rhamnolipid and alginate are required for biofilm formation for protected environment, $P$. aeruginosa is also capable to resist the action of antibiotic by means of efflux pumps and modifying enzymes $[10,11]$.

Motility is among the important virulence features in $P$. aeruginosa due to its crucial role during the initial stage of the infection processes. Chemotaxis-guided search and translocation of $P$. aeruginosa to desirable surfaces are both flagella and pili-aided; allowing the bacterial cells to make contact and attach to those surfaces [12]. This irreversible attachment switches the usual planktonic growth of $P$. aeruginosa into a sessile lifestyle as it prepares for biofilm formation and development [13]. Enclosed in the biofilm structure, the bacterial community is now a thousand-time protected from antibiotic pressure, while continuously attacking the host [14]. Since it is vital for $P$. aeruginosa to succeed this first course, strategically targeting and intervene the motility might be the key for the control and prevention of bacterial infection.

$P$. betle leaves has previously been demonstrated to possess an anti-QS activity against $P$. aeruginosa [15]. It is a tropical creeper plant belongs to the family Piperaceae and its leaf has long been traditionally used for wound healing due to its antiseptic property. Moreover, $P$. betle leaf has been reported for its biological activities such as antidiabetic, antimicrobial, antinociceptive, antioxidant, insecticidal and gastroprotective activities [16]. In this study, we investigated the effects of $P$. betel leaves extract on three different motilities of P. aeruginosa, namely, swimming, swarming and twitching. Swimming is mediated by a single polar flagellum and has been identified as a single cell motion [12]. Twitching, on the other hand, is pili-mediated type of motility while swarming is mediated by both flagella and pili, which both motilities are considered as multicellular type of motions [17]. Intervention at the very early stage of the infection provides us with a considerable advantage over the pathogen, permitting a better management of bacterial infections, particularly infection by $P$. aeruginosa.

\section{MATERIALS AND METHOD}

\subsection{Bacterial strains and subculture conditions}

P. aeruginosa strain 14 (PA14) was used to assess the motility of the pathogen under the influence of a fraction of $P$. betle leaves ethyl acetate extract. PA14 was maintained on King's B medium according to the following recipe; $20 \mathrm{~g}$ peptone, $1.5 \mathrm{~g} \mathrm{KH}_{2} \mathrm{PO}_{4}, 15 \mathrm{~g}$ agar, $15 \mathrm{ml}$ glycerol, $6 \mathrm{ml} 1 \mathrm{M} \mathrm{MgSO}_{4}$ and $\mathrm{ddH}_{2} \mathrm{O}$ to $1 \mathrm{~L}$. PA14 was grown at $37^{\circ} \mathrm{C}$. Autoclaved media was kept in $4^{\circ} \mathrm{C}$ until further use.

\subsection{Fractionation of ethyl acetate extract of $P$. betle leaves}

The ethyl acetate extract of $P$. betel leaves was subjected to preparative thin layer chromatography (TLC) using a silica gel coated-alumina plate to further fraction the crude extract [18]. Solvent system used was a mixture of isopropyl alcohol and chloroform at ratio 1:10. Applied sample was allowed to run in a saturated solvent vapour chamber and separated components were scrutinized under UV light at 254 and $366 \mathrm{~nm}$. TLC plate was cut according to the separation pattern and extract's components were eluted in methanol and dried to recover the intended fractions. Fractions were kept in $-20^{\circ} \mathrm{C}$ until further use and reconstituted in dimethylsulfoxide (DMSO) for the motility assay.

\subsection{Motility assay}

The three types of PA14 motilities (swimming, swarming and twitching) were tested on a semi solid media according to the following recipe per $1 \mathrm{~L}$ preparation; $10.0 \mathrm{~g}$ peptone, $5.0 \mathrm{~g} \mathrm{NaCl}, 3.0 \mathrm{~g}$ yeast extract, with different agar composition for each assay; swim assay: $3.0 \mathrm{~g}$ agar, swarm assay: $7.0 \mathrm{~g}$ agar, twitch assay: $10.0 \mathrm{~g}$ agar [19]. Fraction of ethyl acetate extract of $P$. betle leaves was added to the agar once it was warm upon touch to a final concentration of $100 \mu \mathrm{g} / \mathrm{ml}$. The semi solid agar media were 
air-dried for $3 \mathrm{~h}$ before inoculated with $5 \mu \mathrm{L}$ of PA14 at 1:100 dilution of overnight culture, whereas for twitching assay, PA14 was stabbed to the bottom of the plate. Assay plates were incubated at $37^{\circ} \mathrm{C}$ for $20 \mathrm{~h}$. The diameter of growth was observed for comparison purposes between the treated and untreated assay plate.

\section{RESULTS AND DISCUSSION}

\subsection{Fractionation of ethyl acetate extract of $P$. betle leaves by preparative TLC}

In this study, preparative TLC was performed to separate and isolate bioactive compounds present in $P$. betle leaves extract based on the polarity property of the compounds. Combination of isopropyl alcohol and chloroform as the mobile phase at 1:10 ratio separated the bioactive compounds accordingly, as shown in Figure 1. The TLC plate was then divided into four fractions according to the separation pattern observed under UV light at $254 \mathrm{~nm}$. The polarity of isolated fractions from F1 to F4 are in increasing manner since silica gel is a polar material and would hold on to polar compounds at greater affinity. Thus, non-polar compounds would travel at longer distance (depending on its polarity) along with the mobile phase. The used of preparative TLC in separating and isolating bioactive compounds from a small amount of sample is convenient as it is simple and efficient in terms of cost and time than the column chromatography and the high performance liquid chromatography (HPLC). A semi, non-polar fraction, F2, was chosen to be tested on its effect on motility of PA14 since it showed anti-QS activity in previous study [15].

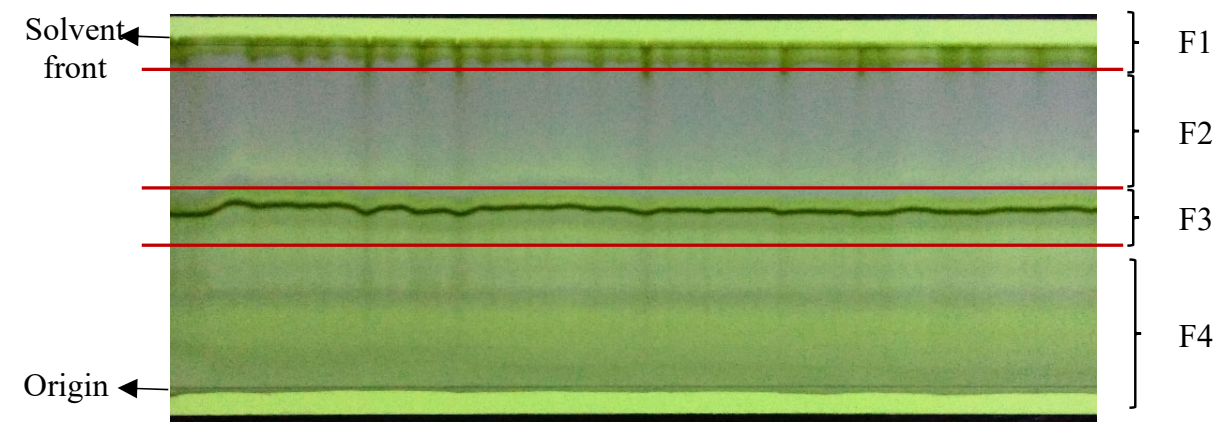

Figure 1: Appearance of preparative TLC plate performed on ethyl acetate extract of $P$. betle leaves as observed under UV light at $254 \mathrm{~nm}$.

\subsection{Effect of $P$. betle leaves fraction on motility of PA14}

P. aeruginosa exhibits three distinct motilities which are swimming, swarming and twitching. These motilities are equally crucial during the first course of infection processes of $P$. aeruginosa; from recognizing appropriate surfaces for attachment to the formation of biofilm for protected environment $[12,13]$. $P$. betle leaves fraction, F2, demonstrated various degree of inhibition against the three type of motilities at concentration of $100 \mu \mathrm{g} / \mathrm{mL}$. Figure 2 shows the motility assay plate appearance after $20 \mathrm{~h}$ of incubation. Among the three motilities, PA14 swimming motility was greatly affected when treated with F2 with a reduction of more than $80 \%$ in the diameter of growth spread as compared to the growth spread of untreated PA14 (Figure 3). Swarming and twitching motilities were less affected with $58 \%$ and 50\% reduction, respectively. In previous work by Datta et. al. (2016), the ethanol extract of $P$. betle leaves were found to reduce the swarming motility by $50.0 \%$ whereas the swimming and twitching motilities, though not as significant, showed a reduction by $7.4 \%$ and $10.7 \%$ respectively [20]. 
Since the extract has previously demonstrated an anti-QS activity against $P$. aeruginosa, the observed defect in the motility could be a unanimous effect of the anti-QS property [15]. On the other hand, the extract could also specifically inhibit the flagella and/or pili of the bacterial cells. Significant reduction in swimming, swarming and twitching strongly suggest that the extract might have affect the mechanism at which the extract exerts on $P$. aeruginosa, such as the biosynthesis and/or the rotation of the flagella and/or pili [17]. The extract might also affect the chemotaxis navigation of the bacterial cells, thus retarding their motility [21]. Another possibility of defect motility could as well be contributed by rhamnolipid that function as a wetting agent to assist the movement of $P$. aeruginosa [22]. With impaired production, it could no longer serve as a lubricant for $P$. aeruginosa to move around. Nonetheless, the adverse effect of the $P$. betle leaves extract on $P$. aeruginosa's motility highlights the effectiveness of the extract in disrupting the initiation of the infection processes and possibly prevents episode of $P$. aeruginosa infection [6]. For a fair data comparison in Figure 3, data were presented in fold-change to that of untreated sample to take into account for any slight variation that might have occurred during the process.

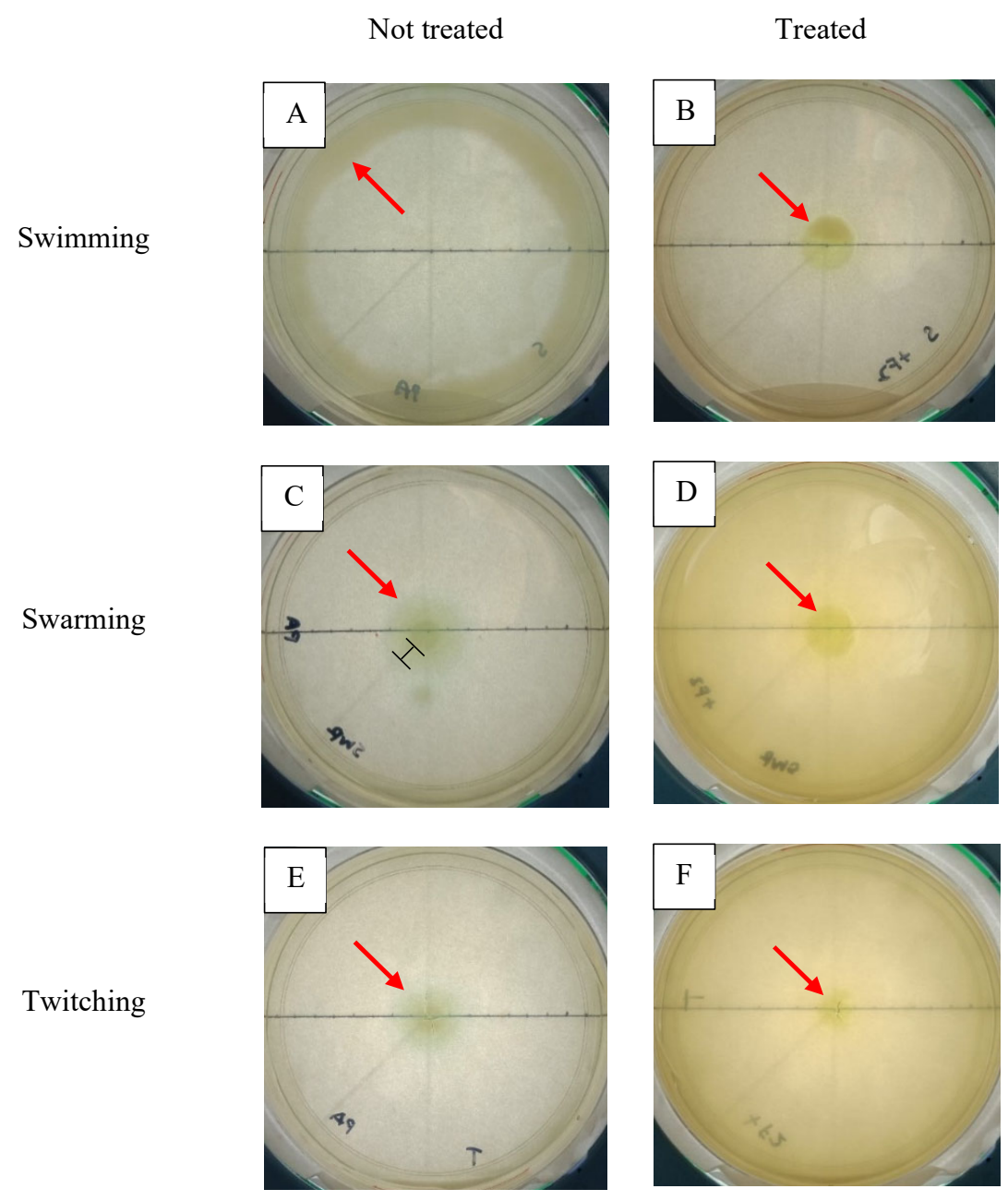

Figure 2: Appearance of motility assay plates with and without treatment of F2. In all three assays, PA14 was inoculated at the middle of the plate and incubated at $37^{\circ} \mathrm{C}$ for $20 \mathrm{~h}$. Edge of growth is indicated by red arrow and the closed-end arrow (C) indicates the width of swarming area of PA14. 


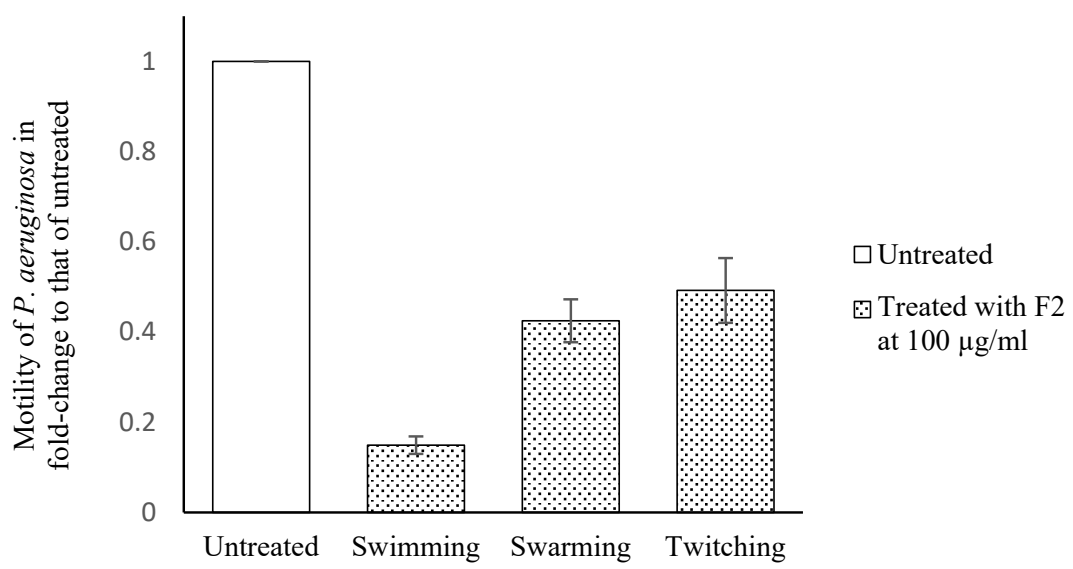

Figure 3: Motility of PA14 as indicated by the growth spread in swimming, swarming and twitching assays. Data presented in fold-change normalized to untreated sample in depicting the inhibition of motility in PA14 by F2

\section{CONCLUSIONS}

This study demonstrated the ability of a fraction of ethyl acetate extract of $P$. betle leaves to adversely affect all three motilities utilized by $P$. aeruginosa, particularly during initiation of infection in a host. Further researches on the negative modulation of the extract on $P$. aeruginosa motility are required to unfold the mechanism involved for an appropriate drug development. Unlike antibiotics, QS inhibition has certainly offered a different perspective in combating bacterial infection by allowing early intervention of the infection processes and thus, permitting a better management of bacterial infections, particularly infection by $P$. aeruginosa. It is hoped with this new mode of action of drug; so called antipathogenic drug; would reduce the damage caused by bacterial infection, while simultaneously put a halt in acquirement of antibiotic resistance by bacterial pathogen.

\section{ACKNOWLEDGMENT}

This study was supported by Universiti Malaysia Pahang under the PGRS180377 and RDU190338 grants.

\section{REFERENCES}

[1] Zambelloni R., Marquez R. and Roe A. J., "Development of anti-virulence compunds: a biochemical review". Chem. Biol. Drug Dis. (85): 43-55. 2015.

[2] Jakobsen T. H. et. al., "Ajoene, a sulfur-rich molecule from garlic, inhibits genes controlled by quorum sensing". Antimicrob. Agents Chemother. (56): 2314-2325. 2012.

[3] Jakobsen T. H. et. al., "Food as a source for quorum sensing inhibitors: iberin from horseradish revealed as a quorum sensing inhibitor of Pseudomonas aeruginosa". Appl. Environ. Microbiol. (78): 2410-21. 2012.

[4] Bobadilla Fazzini R. A, Skindersoe M. E., Bielecki P., Puchalka J., Givskov M. and Martins dos Santos V. A. P., "Protoanemonin: a natural quorum sensing inhibitor that selectively activates iron starvation response”. Environ. Microbiol. (15): 111-120. 2012.

[5] Lee J. and Zhang L., "The hierarchy quorum sensing network in Pseudomonas aeruginosa". Protein Cell (6): 26-41. 2015.

[6] Khan F., Pham D. T. N., Oloketuyi S. F. and Kim Y., "Regulation and controlling the motility properties of Pseudomonas aeruginosa". App. Microb. Biotech. (104): 33-49. 2020. 
[7] Daddaoua A., Fillet S., Fernandez M., Udaondo Z., Krell T. and Ramos J. L., "Genes for carbon metabolism and the ToxA virulence factor in Pseudomonas aeruginosa are regulated through molecular interactions of PtxR and PtxS". PLoS One 7(7): e39390. 2012.

[8] Oglesby A. G., Farrom J. M., Lee J-H., Tomaras A. P., Greenberg E., Pesci E. C. and Vasil M. L., "The influence of iron on Pseudomonas aeruginosa physiology: a regulatory link between iron and quorum sensing system”. J. Biol. Chem. (283):15558-15567

[9] Laarman A. J., Bardoel B. W., Ruyken M., Fernie J., Milder F. J., van Strijp J. A. and Rooijakkers S. H., "Pseudomonas aeruginosa alkaline protease blocks complement activation via the classical and lectin pathways". J. Immunol. (188): 386-393. 2012.

[10] Lequette Y. and Greenberg E. P., "Timing and localization of rhamnolipid synthesis gene expression in Pseudomonas aeruginosa biofilms". J. Bacteriol. (187): 37-44. 2005.

[11] Bonomo R. A. and Szabo D., "Mechanisms of multidrug resistance in Acinetobacter species and Pseudomonas aeruginosa". Clin. Infect. Diseases (43): S49-S56. 2006.

[12] Conrad J. C., Gibiansky M. L., Jin F., Gordon V. D., Motto D. A., Matthewson M. A. et. al., "Flagella and pili-mediated near-surface single-cell motility mechanisms in P. aeruginosa”. Biophys. J. (100): 1608-1616. 2011.

[13] Patriquin G. M., Banin E., Gilmour C., Tuchman R., Greenberg E. P. and Poole K., "Influence of quorum sensing and iron in on twitching motility and biofilm formation in Pseudomonas aeruginosa," J. Bacteriol. (190): 662-671. 2008.

[14] Kostakioti M., Hadjifrangiskou M. and Hultgren S. J., "Bacterial biofilms: development, dispersal, and therapeutic strategies in the dawn of the post-antibiotic era," Cold Spring Harb. Perspect. Med. 3(4): a010306. 2013.

[15] Sedek S. A. M., Latif M. N A., Arifin M. A.and Munaim M. S. A., "Relative assessment of anti-quorum sensing in Piper betle leaves extract via pyoverdin assay". IOP Conf. Ser. Mater. Sci. Eng. (736). 2020.

[16] Arambewela L. S., Arawwawala L. D., Kumaratunga K. G., Dissanayake D. S., Ratnasooriya W. D. and Kumarasingha S. P., "Investigation on Piper betle grown in Sri Lanka”. Pharmacogn Rev. (5): 159-163. 2011.

[17] Nirody J. A., Sun Y. R. and Lo C. J., "The biophysicist's guide to the bacterial flagellar motor". Advances in Physics, (2): 324-343. 2017.

[18] Rabel F. and Sherma J., "Review of the state of the art of preparative thin layer chromatography". J. Liq Chrom. \& Rel. Tech. (40): 165-176. 2017.

[19] Inoue T., Shinggaki R. and Fukui K., "Inhibition of swarmimg motility of Pseudomonas aeruginosa by branched-chain fatty acids". FEMS Microbiol. Letter (281): 81-86. 2008.

[20] Datta S., Jana D., Maity T. R., Samanta A. and Banerjee R., "Piper betle leaf extract affects the quorum sensing and hence virulence of pseudomonas aeruginosa PA01". 3 Biotech 6(1):18. 2016.

[21] Jansari V. H., Potharla V. Y., Riddell G. T. and Bardy S. L., "Twitching motility and cAMP levels: signal transduction through a single methyl-accepting chemotaxis protein". FEMS Microbiol. Letter 363(12):fnw119. 2016.

[22] Blus-Kadosh I, Zilka A., Yerushalmi G. and Banin E., "The effect of pstS and phoB on quorum sensing and swarming motility in Pseudomonas aeruginosa". PLoS One 8(9): e74444. 2013 